Post-print of Rodríguez-Carmona, Escarlata et al. "A novel bio-functional material base on mammalian cell aggresomes" in Applied Microbiology and Biotechnology, September 2015, Volume 99, Issue 17, pp 7079-7088. The final version is available at: DOI: $10.1007 / \mathrm{s} 00253-015-6684-0$

\title{
A novel bio-functional material based on mammalian cell aggresomes
}

Escarlata Rodríguez-Carmona ${ }^{1,3}$, Rosa Mendoza ${ }^{2,3}$, Eugènia Ruiz-Cánovas ${ }^{3, \&}$, Neus FerrerMiralles $^{3,1,2}$, Ibane Abasolo ${ }^{4,2}$, Simó Schwartz $\mathrm{Jr}^{4,2}$, Antonio Villaverde ${ }^{3,1,2}$ and José Luis Corchero $^{2,3,1 *}$

${ }^{1}$ Departament de Genètica i de Microbiologia, Universitat Autònoma de Barcelona, Bellaterra, 08193 Barcelona, Spain.

${ }^{2}$ CIBER de Bioingeniería, Biomateriales y Nanomedicina (CIBER-BBN), Barcelona, Spain.

${ }^{3}$ Institut de Biotecnologia i de Biomedicina, Universitat Autònoma de Barcelona, Bellaterra, 08193 Barcelona, Spain.

${ }^{4}$ CIBBIM-Nanomedicine, Hospital Universitari Vall d'Hebrón and Vall d'Hebrón Institut de Recerca, Universitat Autónoma de Barcelona, 08035 Barcelona, Spain.

\& Present address: Greenaltech; Parc Científic de Barcelona, Edifici Hèlix, c/ Baldiri Reixac 15, 08028 Barcelona

Corresponding author: José Luis Corchero

Institute for Biotechnology and Biomedicine, Universitat Autònoma de Barcelona, Bellaterra, Barcelona 08193, Spain

Phone number: +34935812148

Fax number: +34 935812011

E-mail: jlcorchero@ ciber-bbn.es 


\begin{abstract}
Aggresomes are protein aggregates found in mammalian cells when the intracellular protein degradation machinery is over-titred. Despite they abound in cells producing recombinant proteins of biomedical and biotechnological interest, the physiological roles of these protein clusters and the functional status of the embedded proteins remain basically unexplored. In this work, we have determined for the first time that, like in bacterial inclusion bodies, deposition of recombinant proteins into aggresomes does not imply functional inactivation. As a model,

human $\square$-galactosidase A (GLA) has been expressed in mammalian cells as enzymatically active, mechanically stable aggresomes showing higher thermal stability than the soluble GLA version. Since aggresomes are easily produced and purified, we propose these particles as novel functional biomaterials with potential as carrier-free, self-immobilized catalyzers in biotechnology and biomedicine.
\end{abstract}

Keywords: recombinant protein, protein nanoparticles, immobilized catalyzer, aggresomes. 


\section{Introduction}

Contrarily to what had been previously stated, inclusion bodies (IBs) produced by recombinant bacteria are formed by biologically active polypeptides embedded in amyloid-like networks (Garcia-Fruitos et al. 2005; Tokatlidis et al. 1991; Worrall and Goss, 1989). The unexpected application of IBs as versatile, functional and biocompatible materials paves the road for their use as self-immobilized catalysts when formed by enzymes like reductases (Garcia-Fruitos et al. 2005), oxidases (Nahalka and Nidetzky, 2007; Nahalka et al. 2008a), kinases (Nahalka et al.

2006; Nahalka and Patoprsty, 2009), phosphorylases (Nahalka, 2008) and aldolases (Nahalka et al. 2008b), or as therapeutic agents in protein replacement therapies when formed by therapeutic proteins (Cano-Garrido et al. 2013; Seras-Franzoso et al. 2013a; Seras-Franzoso et al. 2013b; Seras-Franzoso et al. 2014; Vazquez et al. 2012). Mammalian cells instead of bacteria are preferred factories for eukaryotic protein production, because of their ability to perform proper post-translational modifications. In the late 90's, Johnston showed the accumulation of recombinant proteins in subcellular structures of mammalian cells, named "aggresomes" (Johnston et al. 1998). Aggresome formation has been attributed to a cellular response to misfolded, non-proteolyzed proteins that deposit around the microtubule-organizing center (MTOC) by active transport on microtubules. Their formation is prevented by disruption of the dynein-dynactin complex, suggesting that dynein protein is the motor of this transport (Garcia-Mata et al. 1999). Aggresomes show a surrounding cage of intermediate filament (IF) proteins, like vimentin (Johnston et al. 1998), and contain proteasome subunits, ubiquitin and chaperones, consistent with their formation in response to cellular stress. Apart from the main aggregated protein species, aggresomes also contain Hsc70, Hsp40 proteins Hdj1 and Hdj2, and the chaperonin TriC/TCP (Garcia-Mata et al. 1999; Johnston et al. 1998; Wigley et al. 1999). However, the most consistent components of aggresomes are intermediate filament proteins. Even though many efforts have been devoted to reveal formation mechanisms, composition and biological roles of aggresomes (Garcia-Mata et al. 1999; Garcia-Mata et al. 2002; Johnston et al. 1998; Johnston et al. 2000; Junn et al. 2002; Kopito, 2000; Kopito and Sitia, 2000; Wigley et al. 1999), putative functionality and biological activities of the proteins embedded within them have been so far basically unexplored.

In the present study we demonstrate, for the first time, that mammalian cell aggresomes, like in the case of bacterial IBs, retain the biological activity of the forming recombinant protein. By taking the human $\square$-galactosidase A (GLA) as a model, we have obtained fully active and functionally and mechanically stable aggresomes that perform very efficiently in enzymatic processes. These results are discussed in the context of emerging nanostructured materials that when being functional and biocompatible, might have intriguing applications in biomedicine, in order to package functional proteins that, because of their post-translational modifications, cannot be produced in bacteria as functional IBs. 


\section{Material and Methods}

Production of recombinant GLA

The expression vector pOpinE-GLA encodes a full-length version of the human GLA gene, cloned into pOPINE plasmid (Berrow et al. 2007). The suspension-adapted HEK (human embryonic kidney) cell line FreeStyle ${ }^{\mathrm{TM}}$ 293-F (Invitrogen ${ }^{\mathrm{TM}}$, Life Technologies, ref. R790-07) was used to produce GLA by polyethylenimine (PEI)-mediated transient gene expression. Details of GLA production and purification have been previously described (Corchero et al. 2011).

Detection of recombinant GLA in transfected cells

To estimate GLA accumulation into different cellular fractions, 1-mL samples of transfected cells were harvested (3, 4 and 5 days after transfection), centrifuged (15 min, $15000 \mathrm{rpm})$ and supernatants separated from cells. Cell pellets were resuspended in $1 \mathrm{ml}$ of $0.01 \mathrm{M}$ acetic acid $\mathrm{pH} 4.5$ and disrupted by sonication. After centrifugation (15 min, $15000 \mathrm{rpm})$, supernatant containing soluble intracellular fraction was separated, and pellet containing intracellular insoluble fraction was resuspended in $1 \mathrm{ml}$ of $0.01 \mathrm{M}$ acetic acid $\mathrm{pH} 4.5$. All these three S samples were then analyzed by SDS-PAGE and western-blot developed with a rabbit anti-GLA serum (a-gal A H-104: sc-25823, Santa Cruz Biotechnology). Amounts of GLA ineachfraction were estimated by comparison, after SDS-PAGE and western-blot, with known amounts of a recombinantsoluble GLA producedinourlaboratory. Samples and GLA standards were ran in the same gel and processed as a set. Densitometric analyses were performed with Quantity One software (Bio-Rad Laboratories, Inc). Recombinant GLA present in the three fractions (extracellular, intracellular soluble and intracellular insoluble) were used to estimate the "Total" recombinant GLA produced (taken as $100 \%$ of recombinant protein produced), and then the values from each fraction were compared to this "Total" value to obtain the percentages shown inTable1.

Aggresome formation was also checked by transmission electronic microscopy (TEM). For that, a total of $\square 10^{7}$ cells were taken beforetransfection(as negativecontrol)and at different times post-transfection. After centrifugation (5 min, $1200 \mathrm{rpm}$ ), cell pellets were resuspended in $2.5 \mathrm{ml}$ of fixing solution (paraformaldehyde 2\%, glutaraldehyde 2.5\%). After fixation (15 min, at room temperature), samples were stored at $4^{\circ} \mathrm{C}$ until TEM analysis. Samples were observed in a JEM-1400 transmission electron microscope (Jeol) with a CCD GATAN ES1000W Erlangshen camera.

Aggresome purification 
At 5 days post-transfection, the soluble, secreted GLA present in the culture medium was separated from cells by centrifugation $(15 \mathrm{~min}, 15000 \mathrm{rpm})$ of transfected cultures. Supernatants were removed and cell pellets were used to purify aggresomes. The protocol and "disrupting buffers" previously described (Garcia-Mata et al. 1999) were used for cell lysis. Briefly, cell pellets were resuspended with either $2 \%$ Triton X-100 in PBS, IPB buffer $(10 \mathrm{mM}$ Tris-HCl, $\mathrm{pH}$ 7.5, $5 \mathrm{mM}$ EDTA, $1 \%$ NP-40, $0.5 \%$ deoxycholate, $150 \mathrm{mM} \mathrm{NaCl}$ ), or RIPA buffer (50 mM Tris$\mathrm{HCl}, \mathrm{pH} 8,1 \% \mathrm{NP}-40,0.5 \%$ deoxycholate, $0.1 \%$ SDS, $150 \mathrm{mM} \mathrm{NaCl}$ ). Since GLA is a lysosomal enzyme (with optimal activity at $\mathrm{pH} 4.5$ ), it is likely that incubation in an acidic buffer would better preserve its enzymatic activity than IPB or RIPA buffers (with higher $\mathrm{pH}$, deleterious for GLA enzymatic activity). Therefore, a fourth "disrupting buffer" was included: IPB buffer was modified by replacing $10 \mathrm{mM}$ Tris- $\mathrm{HCl}$ with $0.01 \mathrm{M}$ acetic acid at $\mathrm{pH} 4.5$. Thus, the final "acetic acid disrupting buffer" composition was $0.01 \mathrm{M}$ acetic acid at $\mathrm{pH} 4.5$, supplemented with $5 \mathrm{mM}$ EDTA, $1 \%$ NP-40, $0.5 \%$ deoxycholate, and $150 \mathrm{mM} \mathrm{NaCl}$. All disrupting buffers were supplemented with a protease inhibitor cocktail (Complete EDTA-free, Roche). Cells were resuspended in the corresponding buffer, incubated for $30 \mathrm{~min}$ on ice, and then passed 10 times through a 25-gauge needle. Soluble and insoluble intracellular fractions were separated by centrifugation $\left(6,000 \mathrm{rpm}, 2 \mathrm{~min}, 4^{\circ} \mathrm{C}\right)$. Supernatants were eliminated, and pellets containing aggresomes were washed with acetic acid $0.01 \mathrm{M}, \mathrm{pH}$ 4.5. After wash, GLA aggresomes were centrifuged $\left(6,000 \mathrm{rpm}, 2 \mathrm{~min}, 4^{\circ} \mathrm{C}\right)$, resuspended again in acetic acid buffer (usually 20 -fold concentrated respect to initial cell culture volume), aliquoted and stored at $-80^{\circ} \mathrm{C}$ until tested.

To compare amounts of aggresomes recovered by using different disrupting buffers, equal volumes of purified aggresomes were analyzed by SDS-PAGE and further Coomasie staining and western-blot.

Characterization of recombinant GLA aggresomes

Enzymatic activity of soluble GLA and aggresomes was assayed, in vitro, fluorometrically as described by Desnick (Desnick et al. 1973) and Mayes (Mayes et al. 1981). Briefly, activity was assayed using as substrate 4-methylumbelliferyl- $\alpha$-D-galactoside (4MUG, Sigma Chemical), at $2.46 \mathrm{mM}$ in assay buffer (0.01 $\mathrm{M}$ acetic acid, $\mathrm{pH}$ 4.5). Reaction mixtures contained $100 \mu \mathrm{l}$ substrate and $25 \mu$ l enzyme sample, in which purifiedaggresomes were dilutedinaceticacid in order to assure readings falling within the standard curve range. Reactions took place in agitation $\left(37^{\circ} \mathrm{C}, 1\right.$ hour), and were stopped with $1.25 \mathrm{~mL}$ of $0.2 \mathrm{M}$ glycine- $\mathrm{NaOH}(\mathrm{pH} 10.4)$. The released product (4-methylumbelliferone, 4-MU) was determined by fluorescence measurement at 365 and $450 \mathrm{~nm}$ as excitation and emission wavelengths, respectively. Samples of commercial product 4-MU (Sigma Chemical) ranging from 5 to $500 \mathrm{ng} / \mathrm{mL}$ in $0.2 \mathrm{M}$ glycine- $\mathrm{NaOH}$ buffer, $\mathrm{pH}$ 10.4, were used to obtain a calibration curve in order totransform fluorescence readings into product 4-MUconcentration. Specific enzymatic activities are expressed as $\mu \mathrm{mol}$ 4-MU/h/mg protein. 
To identify the main proteins detected by Coomasie staining in purified aggresomes, their corresponding bands were cut out from the stained gels and trypsin digested. The resulting peptides were analyzed (by "peptide mass fingerprint") by matrix-assisted laser desorption/ionization (time-of-flight), MALDI-TOF, and their sequences compared to SwissProt 2011_03 database.

Size distribution of aggresomes was measured by dynamic light scattering (DLS) at $633 \mathrm{~nm}$ wavelength, combined with non-invasive backscatter technology (NIBS) in a DLS analyzer (Zetasizer Nano ZS, Malvern Instruments Limited, Malvern, U.K.). Dispersions of purified aggresomes were prepared by sonication ( $2 \mathrm{~min}, 0.5$ seconds on/off cycles, room temperature), diluted in acetic acid buffer, and measured at $20^{\circ} \mathrm{C}$ without filtering. Mean value of three measurements was taken as the hydrodynamic aggresome diameter. The software used to analyze the data is derived from a deconvolution of the measured intensity autocorrelation function of the sample, using a non-negatively constrained least squares (NNLS) fitting algorithm (software provided by Malvern with the Zetasizer instrument). The "Meanintensity" of the peak obtained (instead of the $\mathrm{z}$-average number) was used as an estimation of the aggresomes diameter (in $\mathrm{nm}$ ), since the $\mathrm{z}$-average mean size can be sensitive to the presence

of even small populations of big aggregates.

Purified aggresomes were also checked by scanning electronic microscopy (SEM). For that, they were deposited on Nuclepore $\square$ Track-Etched polycarbonate membranes with pore size of $0.2 \mu \mathrm{m}$ (Whatman Ltd., United Kingdom). Then, samples were fixed with $2.5 \%$ glutaraldehyde in $0.1 \mathrm{M}$ phosphate buffer ( $\mathrm{PB}, \mathrm{pH} 7.4)$, washed 4 times (10 min each) in $0.1 \mathrm{M} \mathrm{PB}$, post-fixed in $1 \%$ (wt/vol) osmium tetraoxide with $0.7 \%$ ferrocyanide in $\mathrm{PB}$, washed in water, dehydrated in an ascending ethanol series $(50,70,80,90$, and $95 \%$ for $10 \mathrm{~min}$ each and twice with $100 \%$ ethanol), and dried by critical point drying with $\mathrm{CO}_{2}$. Samples were mounted on adhesive carbon films and coated with gold. Images were taken with an EVO® MA 10 scanning electron microscope (Zeiss) at an accelerating voltage of $20 \mathrm{kV}$ and an EDS Oxford INCA detector.

Spontaneous release of GLA from aggresomes

The putative spontaneous release of GLA enzyme from aggresomes when incubated at $37^{\circ} \mathrm{C}$ (that could interfere in reusability experiments) was checked. For that, aggresomes were incubated at $37^{\circ} \mathrm{C}$ in agitation. At different time-points, samples were taken and centrifuged (15 min, $15000 \mathrm{rpm})$ in order to separate aggresomes from solubilized GLA that could have been released to the supernatant. After centrifugation, supernatants were separated and remaining aggresomes resuspended in their original volume with $0.01 \mathrm{M}$ acetic acid, $\mathrm{pH}$ 4.5. Finally, enzymatic activity and western-blot of aggresomes and supernatants were performed. 
Thermal stability of soluble GLA and aggresomes

Soluble GLA and aggresomes were diluted in $0.01 \mathrm{M}$ acetic acid, $\mathrm{pH} 4.5$, aliquoted and incubated at $37^{\circ} \mathrm{C}$. At different time periods, sampleswere taken and directly submitted to the enzymatic activity test. In the aggresome samples, putative solubilized/released enzyme and enzyme still in the aggregated state were not separated, meaning that measured activities were the summary of those from both released and aggregated enzyme. Activity of the aliquots was compared to that determined for aliquots kept always at $-80^{\circ} \mathrm{C}$, used as a reference.

Reusability of GLA aggresomes

Before testing aggresomes reusability, they were incubated for $1 \mathrm{~h}$ at $37^{\circ} \mathrm{C}$, in agitation. Then, aggresomes were centrifuged (15 $\mathrm{min}, 15000 \mathrm{rpm})$ to discard GLA spontaneously released to the supernatant. Aggresomes were resuspended in their original volume with $0.01 \mathrm{M}$ acetic acid, $\mathrm{pH}$ 4.5. After that, reusability was evaluated by performing successive enzymatic activity measurements as described before. After each reaction cycle, aggresomes were recovered by centrifugation (15 min, $15000 \mathrm{rpm})$. Supernatants containing the reaction product were transferred to a tube with $1.25 \mathrm{ml}$ of $0.2 \mathrm{M}$ glycine-NaOH buffer ( $\mathrm{pH}$ 10.4). The amount of product released was determined as mentioned before. Aggresomes were washed with assay buffer and resuspended again in $25 \mu \mathrm{l}$. Then, fresh substrate was added and next measurement was carried out and compared with the first round (defined as 100\%). 


\section{Results}

GLA expression and formation of aggresomes

Expression and accumulation of GLA into the extracellular, and soluble and insoluble intracellular fractions were checked by SDS-PAGE and western-blot, showing a progressive accumulation of GLA into these three cellular fractions (Figure 1). At day 5, the total, accumulated GLA reached $>160 \mu \mathrm{g} / \mathrm{ml}$. From that, $\square 50 \%$ corresponded to the soluble from, secreted form of the enzyme, while the rest remained intracellular, either as soluble $(10.6 \%)$ or insoluble versions $(40 \%)$ (Table 1). Intracellular protein shows an apparent molecular weight slightly lower than that of the secreted form (Figure 1). Trypsin digestion of the band corresponding to GLA embedded into aggresomes and peptide mass fingerprint identified 12

sequences matching GLA theoretical sequence. From such 12 sequences, two of them (MQLRNPELHLGCALALR and NPELHLGCALALR) were located within the GLA signal peptide (residues 1-17 and 5-17, respectively).

GLA expression and accumulation into aggresomes were visualized by TEM (Figure 2). In contrast to images from cells before transfection (Figure 2, a and b), images taken at 3 and 5 days post-transfection (Figure 2, c to f) revealed clearly visible aggregates throughout the cytoplasm, not present before transfection. Since all samples used to obtain TEM images (including cells before transfection) were treated simultaneously, with the same protocol and reagents, and moreover cytoplasmic protein aggregates are only $\underline{\text { seen after transfection (and therefore, after GLA expression), the structures identified as }}$ aggresomes are not expected to be an experimental artifact._Such aggregates are not homogeneous, but formed by the self-assembly of smaller, repeated building blocks, consisting on spherical structures of $\square 50-100 \mathrm{~nm}$ in diameter.

Purification of aggresomes

The amount and purity of recovered aggresomes was determined by SDS-PAGE and westernblot. According to densitometric analysis of membranes, samples obtained with RIPA buffer contained the lowest GLA amounts, while the use of "acetic acid disrupting buffer" allowed to recover 1.3-, 1.5- and 2-fold more recombinant protein within aggresomes than that found with Triton, IPB and RIPA buffers, respectively. Presence of solubilizing agents, like SDS in RIPA buffer, could explain this fact, since some of the protein initially entrapped within aggresomes could be solubilized (and therefore lost from the insoluble fraction) during the purification process. Irrespective of the buffer used, protein patterns within such structures are essentially the same (not shown), suggesting that disruption buffer does not significantly affect aggresome purity and composition. 
Enzymatic and architectonic characterization of aggresomes

Since large protein aggregates are typically monitored at $350-360 \mathrm{~nm}$, and in order to avoid any interference in the enzymatic assay readings due to the presence of such aggregates, an absorbance spectra control (in the range of 300 to $450 \mathrm{~nm}$ ) of 100-fold diluted aggresomes was performed, resulting in absorbance values $<0.1$. Therefore, no interference from aggresomes is expected when performing the GLA enzymatic assay. Performing the GLA enzymatic assay using GLA aggresomes as sample showed that aggregation and incorporation of GLA into aggresomes did not result into enzymatic inactivation, as shown in Table 2. Aggresomes purified in "acetic acid disrupting buffer" clearly showed the highest specific activity, which is not surprising since $\mathrm{pH}$ of this buffer is optimal for GLA activity. The specific activities for the more active aggresomes and for soluble GLA (Corchero et al. 2011) were 1,095 $\square 33$ and 1,947 $\square 259$ $\mu \mathrm{mol} / \mathrm{h} / \mathrm{mg}$ protein, respectively. That means that GLA incorporated into aggresomes retains $\square 56 \%$ of the specific activity of its soluble counterpart. Taking into consideration these results, we chose the "acetic acid disrupting buffer" to purify functional aggresomes used next.

Size of aggresomes was determined by DLS. When cheking the distribution of sizes of our samples, results showedinallsamples the presence ofone mainpeak (correspondingtomore than9095\% ofthe sampleintensity), and then insomecases, the presenceofsomeimpurities of highersizes, but corresponding to very small populations (less than 5-10\%) in the sample (data not shown). Regarding the number of decays in the auto-correlation functions, our data performedalways giving aunique, singleexponentialdecayfunction, indicative ofthe presence of amainpopulation. After thissingledecay,signalshowedinsomecasessomescatteringvery near tothe Xaxis, probablyindicative of(and supporting)the presenceofthe abovementioned impurities in the form of "big" aggregates. The polydispersityindexes obtainedwere $0.446+1-$

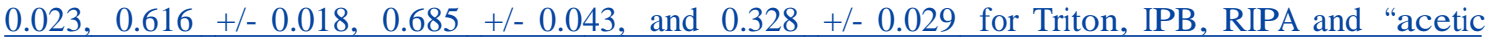
acid" disrupting buffers respectively, indicative that samples are suitable for the dynamic light scattering(DLS)analysis.

Aggresomes purified by using the disrupting buffers previously described (Garcia-Mata et al. 1999) showed sizes around $1 \mu \mathrm{m}$ of diameter: $792+/-111,1037+/-38$ and $915+/-97 \mathrm{~nm}$ for Triton, IPB and RIPA buffers, respectively (Table 2), in agreement with TEM observations (Figure 2e and 2f). Interestingly, DLS determination of aggresomes purified in "acetic acid disrupting buffer" resulted in higher values (1698 +/- $29 \mathrm{~nm}$ in diameter), in agreement with SEM images (Figure 3).

The spherical building blocks detected in TEM images were also clearly visible by SEM (see Figure 3b), confirming the "aggregate of aggregates" architecture of aggresomes. Structures

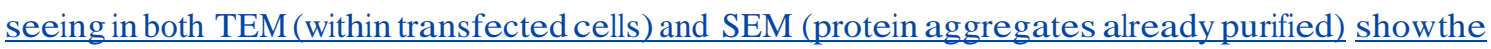
same architecture: they seem to be formed by the coalescence of small aggregates 
(ofabout 50nm), whichisdistinctiveofaggresomes. However, the building blocks seen in SEM are not as tightly packed that those seen in TEM (compare Figure $2 \mathrm{f}$ with Figure $3 \mathrm{~b}$ ).

Compositional analysis of aggresomes

Analysis of purified aggresomes by SDS-PAGE and further Coomasie staining showed that such protein aggregates are not completely pure. On the contrary, some other cell proteins coaggregate with recombinant GLA during aggresomes formation, appearing later as contaminants in purifiedaggresomes. This allowed us to determine the nature of some of such contaminant proteins by cutting off from the stained gel the bands corresponding to these main contaminants. MALDI-TOF analysis was performed on the four predominant proteins (according to Coomasie staining) found in purified aggresomes. Results confirmed that one of these bands corresponded to human GLA enzyme or its precursor. Finding GLA precursor within aggresomes was not surprising, since some unprocessed GLA molecules still containing the secretion peptide might be trapped within aggresomes. Chaperone Hsp70 was found associated to aggresomes in significant amounts. Also, tubulin (a protein marker for MTOC) and actin (the monomeric subunit of the cytoskeleton microfilaments) are important components of aggresomes.

Spontaneous solubilization of aggresomes

During the first hour of incubation at $37^{\circ} \mathrm{C}, \sim 20-25 \%$ of the GLA initially entrapped within aggresomes was released to the supernatant, indicative of a spontaneous protein solubilization and release. Afterwards, the aggresomal fraction remained basically constant. This was confirmed by both the decrease of enzymatic activity in the insoluble fraction (Fig. 4a) in parallel with the appearance of activity in the supernatant (corresponding to solubilized enzyme) (Fig. 4b). Enzyme solubilization during the first hour was also confirmed by western-blot (Fig. 4c and 4d).

Stability and reusability of aggresomes

Thermal stabilities of soluble GLA and aggresomes were compared by following their enzymatic activity after incubation in acetic acid buffer, at $37^{\circ} \mathrm{C}$. A clearly higher stability of aggresomes was observed (Fig. 5a), as the activity of soluble GLA decreased rapidly, with less than $5 \%$ of activity remaining after 1 day. However, for aggresomes, $68 \%$ and $79 \%$ of activity remained after 7 and 14 days, respectively. These results indicate that GLA embedded within the aggresomes is "protected" and more resistant to thermal inactivation than its soluble counterpart. According to our results, soluble GLA is very sensitive to heat-inactivation. Therefore, putative released GLA from aggresomes, even if not separated from aggresomes 
beforetesting, is not expected tointerferesignificantlyinthe finalenzymaticactivityfoundfor

aggresomes, especiallyafterseveraldaysat $37^{\circ} \mathrm{C}$.

Regarding reusability (determined by successive enzymatic assays after aggresomes recovery from the reaction mixture by centrifugation), aggresomes are easily recovered and, according to the results, could be reused at least in 5 consecutive enzymatic assays. A $70 \%$ of enzymatic activity still remained after 5 rounds (Fig. 5b). Sinceaggresomeshad beenincubated1hour at

$\underline{37^{\circ} \mathrm{C} \text { and the spontaneously GLA released eliminated by centrifugation before starting the }}$ reusabilityexperiments, this drop in the enzymatic activity along consecutive assays is probably due to loss of aggresomes during centrifugation steps. 


\section{Discussion}

Through the production of a model GLA in recombinant mammalian cells, we have demonstrated, for the first time, that mammalian cell aggressomes retain at significant extent the biological activity of the forming protein, similarly to what had been observed in bacterial IB (Garcia-Fruitos et al. 2005; Garcia-Fruitos et al. 2007). Contrarily to the conventional view on these protein particles, this observation indicates that these protein clusters are not exclusively composed by misfolded proteins but probably by functional folding intermediates or by a mixture of misfolded and properly folded proteins. Since GLA incorporated into aggresomes shows

$\square 56 \%$ of the specific activity of its soluble counterpart, the possibility of residual activity by cross-contamination with soluble enzyme during purification should be discarded. Interestingly, although it has been never quantitatively determined, GFP aggresomes are fluorescent (GarciaMata et al. 1999; Garcia-Mata et al. 2002; Shimohata et al. 2002), indicating that the GLA activity determined in the present study is not a peculiar case.

According to solubilization experiments, around 20-25\% of the GLA initially entrapped within aggresomes is solubilized and released to the supernatant under mild conditions (as shown in Fig. 4). The protein solubilized from aggresomes at early incubation times shows poor thermal stability, and become heat-inactivated (Fig. 4b) soon after its release from the aggresome. The enzyme still remaining within aggresomes after 1 hour of incubation seems to form a core highly resistant to solubilization. This "bimodal" architecture of aggresomes might be functionally similar to that proposed for bacterial IBs, in which a fraction of functional releasable protein coexists with mechanically stable, non-functional versions of the same protein (Cano-Garrido et al. 2013). Probably related to such conformational heterogeneity, GLA aggregates are formed

by the assembly of smaller, repeated building blocks consisting on spherical structures of $\square 50$ $100 \mathrm{~nm}$ in diameter (Fig. 3). This aggresome architecture, called "aggregate of aggregates", is in agreement with previous reports (Garcia-Mata et al. 1999; Ioannou et al. 1992; Kopito, 2000; Kopito and Sitia, 2000). According to this model, aggresome building blocks are produced throughout the cytoplasm by polypeptide coaggregation into single aggresomal particles or building blocks. In cells, such particles have a regular size (Garcia-Mata et al. 1999; Johnston et al. 2000), supporting the idea that a fixed number of peptides aggregate form these building blocks. After their formation, these particles are transported to the MTOC, where they incorporate into the final, single aggresome. Electron microscopy has confirmed that building particles are loosely associated with each other and do not coalesce (Garcia-Mata et al. 1999; Johnston et al. 1998). A similar model have been described for IBs formation in E. coli: for example, expression of asparaginase resulted in the formation, within an individual cell, of a high number of small aggregates which further loosely associate amongst themselves to form bigger aggregates (Upadhyay et al. 2012). 
Analysis of purifiedaggresomesshowed that such proteinaggregatesare notcompletelypure. On the contrary, some impurities are co-purified with aggregated recombinant GLA. The presence of such contaminants that co-aggregate within eukaryotic aggresomes is not unexpected and has been previously described in the literature. Chaperone Hsp70 was found associated to aggresomes. In this regard, it has been described the Hsp70 role in promoting aggresome formation (Zhang and Qian, 2011), based on the Hsp70 interaction with the cochaperone ubiquitin ligase carboxyl terminal of Hsp70/Hsp90 interacting protein (CHIP). Disruption of such Hsp70-CHIP interaction prevents aggresome formation, whereas a dominantnegative CHIP mutant sensitizes the aggregation of misfolded protein. In another study, Wang described the presence of Hsp70 associated to polyQ aggregates produced in yeast (Wang et al. 2007). Also, tubulin and actin are important components of aggresomes. The presence of cytoskeleton-associated proteins in aggresomes is not surprising: the large size of mammalian cells, together with the presence of the cytoskeleton, a structure that could restrict the motility of particulate material (Gershon et al. 1985), implies that non-difussional mechanisms are needed to sequester aggregates within the cytoplasm and transport them to the MTOC. For that purpose, formation of aggresomes must exploit the transport on microtubules (Garcia-Mata et al. 1999; Johnston et al. 2002; Kopito, 2000; Kopito and Sitia, 2000), resulting in the fact that cytoskeleton-related proteins, like vimentin, tubulin, actin and others, are usually found intimately associated with aggresomes. It has been shown that small aggregates generated at the cell periphery are transported within the cytoplasm at a rate of $0.32 \mu \mathrm{m} / \mathrm{min}$ towards the aggresome (Garcia-Mata et al. 1999). A model for microtubule-based delivery of protein aggregates to aggresomes has been described (Kopito, 2000; Kopito and Sitia, 2000). In agreement with our results, it has been described the presence of $\square$-tubulin, as an aggresomerelated protein in $\square$-synuclein-containing aggresomes (Chiba et al. 2012) and in perinuclear aggregate bodies formed by a truncated protein containing expanded polyQ stretches

(Shimohata et al. 2002). All these studies corroborate our findings about the presence of Hsp70 chaperone, actin and tubulin as aggresomes-related proteins.

Finally, aggresomes showed protection of the sequestered form of the enzyme within the aggresome fraction towards thermalinactivation in solution, and in our testing system could be reused at least up to 5 rounds of consecutive enzymatic assays (Fig. 5).

The proof-of-concept shown here of aggresomes as immobilized biofunctional particles could be in the line of the development of a new type of biomaterial, similarly to the application of bacterial IBs as protein release nanoparticles in tissue engineering and substitutive therapies (Seras-Franzoso et al. 2013a; Seras-Franzoso et al. 2013b; Seras-Franzoso et al. 2014). Since some eukaryotic proteins are not produced in their active form when expressed in prokaryotic systems, mammalian aggresomes could be an alternative to package functional proteins in mechanically stable, protein releasing nano-microstructured materials. GLA glycosylation itself has been described as crucial to the enzyme activity (Ioannou et al. 1998), hampering their 
production in bacterial expression systems. In this context, it has been possible to adapt a purification protocol for aggresomes to the peculiar functional demands of aggresomes (mainly its optimal $\mathrm{pH}$ for catalytic activity). In summary, since mammalian cell aggresomes retain the enzymatic activity of their protein components, show improved stability versus soluble protein versions, and can be purified by function-friendly protocols, we propose these structures as a novel type of carrier-free, self-assembled immobilized biomaterial, with putative applications in biotechnology and biomedicine. This strategy could be of special interest for those proteins that, like GLA, cannot be expressed in prokaryotic systems. The model GLA used in this work formed enzymatically active aggresomes, representing a proof-of-concept for such aggregates as a new type of carrier-free, self-assembled immobilized biomaterial. However, in addition, $\square-$ galactosidases are also of particular interest regarding biotechnological and biomedical applications, and GLA aggresomes could have putative applications in both biotechnological (such as the removal of raffinose oligosaccharides from several legume-based foods) and biomedical fields (such as improved methods to deliver enzymatically active GLA in the field of innovative enzyme replacement therapies in Fabry disease patients). 
Acknowledgments:

MALDI-TOF analyses were carried out in the Proteomics and Structural Biology facility (SepBioEs) from UAB, member of ProteoRed network. We thank the Protein Expression Core Facility, Institute for Research in Biomedicine (IRB, Barcelona) for cloning the GLA gene into the pOPIN vector. We appreciate the technical support from the UAB Scientific and Technical Services SCAC (Servei de Cultius Cel-lulars, Producció d'Anticossos i Citometria). Protein production has been partially performed by the ICTS "NANBIOSIS", more specifically by the Protein Production Platform of CIBER in Bioengineering, Biomaterials \& Nanomedicine (CIBERBBN)/ IBB, at the UAB (http://www.ciber-bbn.es/en/programas/89-plataforma-de-produccion-deproteinas-ppp). Authors appreciate the financial support through the grant EUI2008-03610 (MICINN) linked to the ERANET-IB 08-007 project, from Agència de Gestió d'Ajuts Universitaris i de Recerca (2014SGR-132), and for the grant to the project "Development of nanomedicinas for enzymatic replacement therapy in Fabry disease", granted by the Fundació Marató TV3. We also acknowledge the support of the CIBER de Bioingeniería, Biomateriales y Nanomedicina (CIBER-BBN), an initiative funded by the VI National R\&D\&i Plan 2008-2011, Iniciativa Ingenio 2010, Consolider Program, CIBER Actions and financed by the Instituto de Salud Carlos III with assistance from the European Regional Development Fund. AV was distinguished with an ICREA ACADEMIA award (Catalonia, Spain).

Conflict of interest:

The authors declare no commercial or financial conflict of interest. 
Reference List

Berrow NS, Alderton D, Sainsbury S, Nettleship J, Assenberg R, Rahman N, Stuart DI, Owens RJ. (2007). A versatile ligation-independent cloning method suitable for high-throughput expression screening applications. Nucleic Acids Res 35:e45.

Cano-Garrido O, Rodriguez-Carmona E, ez-Gil C, Vazquez E, Elizondo E, Cubarsi R, SerasFranzoso J, Corchero JL, Rinas U, Ratera I, Ventosa N, Veciana J, Villaverde A, Garcia-Fruitos E. (2013). Supramolecular organization of protein-releasing functional amyloids solved in bacterial inclusion bodies. Acta Biomater 9:6134-6142.

Chiba Y, Takei S, Kawamura N, Kawaguchi Y, Sasaki K, Hasegawa-Ishii S, Furukawa A, Hosokawa M, Shimada A. (2012). Immunohistochemical localization of aggresomal proteins in glial cytoplasmic inclusions in multiple system atrophy. Neuropathol Appl Neurobiol 38:559-571.

Corchero JL, Mendoza R, Lorenzo J, Rodriguez-Sureda V, Dominguez C, Vazquez E, FerrerMiralles N, Villaverde A. (2011). Integrated approach to produce a recombinant, his-tagged human alpha-galactosidase a in mammalian cells. Biotechnol Prog 27:1206-1217.

Desnick RJ, Allen KY, Desnick SJ, Raman MK, Bernlohr RW, Krivit W. (1973). Fabry's disease: enzymatic diagnosis of hemizygotes and heterozygotes. Alpha-galactosidase activities in plasma, serum, urine, and leukocytes. J Lab Clin Med 81:157-171.

Garcia-Fruitos E, Aris A, Villaverde A. (2007). Localization of functional polypeptides in bacterial inclusion bodies. Appl Environ Microbiol 73:289-294.

Garcia-Fruitos E, Gonzalez-Montalban N, Morell M, Vera A, Ferraz RM, Aris A, Ventura S, Villaverde A. (2005). Aggregation as bacterial inclusion bodies does not imply inactivation of enzymes and fluorescent proteins. Microb Cell Fact 4:27.

Garcia-Mata R, Bebok Z, Sorscher EJ, Sztul ES. (1999). Characterization and dynamics of aggresome formation by a cytosolic GFP-chimera. J Cell Biol 146:1239-1254. 
Garcia-Mata R, Gao YS, Sztul E. (2002). Hassles with taking out the garbage: aggravating aggresomes. Traffic 3:388-396.

Gershon ND, Porter KR, Trus BL. (1985). The cytoplasmic matrix: its volume and surface area and the diffusion of molecules through it. Proc Natl Acad Sci U S A 82:5030-5034.

Ioannou YA, Bishop DF, Desnick RJ. (1992). Overexpression of human alpha-galactosidase A results in its intracellular aggregation, crystallization in lysosomes, and selective secretion. J Cell Biol 119:1137-1150.

Ioannou YA, Zeidner KM, Grace ME, Desnick RJ. (1998). Human alpha-galactosidase A: glycosylation site 3 is essential for enzyme solubility. Biochem J 332 ( Pt 3):789-797.

Johnston JA, Dalton MJ, Gurney ME, Kopito RR. (2000). Formation of high molecular weight complexes of mutant $\mathrm{Cu}, \mathrm{Zn}$-superoxide dismutase in a mouse model for familial amyotrophic lateral sclerosis. Proc Natl Acad Sci U S A 97:12571-12576.

Johnston JA, Illing ME, Kopito RR. (2002). Cytoplasmic dynein/dynactin mediates the assembly of aggresomes. Cell Motil Cytoskeleton 53:26-38.

Johnston JA, Ward CL, Kopito RR. (1998). Aggresomes: a cellular response to misfolded proteins. J Cell Biol 143:1883-1898.

Junn E, Lee SS, Suhr UT, Mouradian MM. (2002). Parkin accumulation in aggresomes due to proteasome impairment. J Biol Chem 277:47870-47877.

Kopito RR. (2000). Aggresomes, inclusion bodies and protein aggregation. Trends Cell Biol 10:524-530.

Kopito RR, Sitia R. (2000). Aggresomes and Russell bodies. Symptoms of cellular indigestion? EMBO Rep 1:225-231.

Mayes JS, Scheerer JB, Sifers RN, Donaldson ML. (1981). Differential assay for lysosomal alpha-galactosidases in human tissues and its application to Fabry's disease. Clin Chim Acta $112: 247-251$ 
Nahalka J. (2008). Physiological aggregation of maltodextrin phosphorylase from Pyrococcus furiosus and its application in a process of batch starch degradation to alpha-D-glucose-1phosphate. J Ind Microbiol Biotechnol 35:219-223.

Nahalka J, Dib I, Nidetzky B. (2008a). Encapsulation of Trigonopsis variabilis D-amino acid oxidase and fast comparison of the operational stabilities of free and immobilized preparations of the enzyme. Biotechnol Bioeng 99:251-260.

Nahalka J, Gemeiner P, Bucko M, Wang PG. (2006). Bioenergy beads: a tool for regeneration of ATP/NTP in biocatalytic synthesis. Artif Cells Blood Substit Immobil Biotechnol 34:515-521.

Nahalka J, Nidetzky B. (2007). Fusion to a pull-down domain: a novel approach of producing Trigonopsis variabilisD-amino acid oxidase as insoluble enzyme aggregates. Biotechnol Bioeng $97: 454-461$

Nahalka J, Patoprsty V. (2009). Enzymatic synthesis of sialylation substrates powered by a novel polyphosphate kinase (PPK3). Org Biomol Chem 7:1778-1780.

Nahalka J, Vikartovska A, Hrabarova E. (2008b). A crosslinked inclusion body process for sialic acid synthesis. J Biotechnol 134:146-153.

Seras-Franzoso J, Peebo K, Corchero JL, Tsimbouri PM, Unzueta U, Rinas U, Dalby MJ, Vazquez E, Garcia-Fruitos E, Villaverde A. (2013a). A nanostructured bacterial bioscaffold for the sustained bottom-up delivery of protein drugs. Nanomedicine (Lond).

Seras-Franzoso J, Peebo K, Garcia-Fruitos E, Vazquez E, Rinas U, Villaverde A. (2014). Improving protein delivery of fibroblast growth factor- 2 from bacterial inclusion bodies used as cell culture substrates. Acta Biomater 10:1354-1359.

Seras-Franzoso J, Steurer C, Roldan M, Vendrell M, Vidaurre-Agut C, Tarruella A, Saldana L, Vilaboa N, Parera M, Elizondo E, Ratera I, Ventosa N, Veciana J, Campillo-Fernandez AJ, Garcia-Fruitos E, Vazquez E, Villaverde A. (2013b). Functionalization of 3D scaffolds with protein-releasing biomaterials for intracellular delivery. J Control Release 171:63-72. 
Shimohata T, Sato A, Burke JR, Strittmatter WJ, Tsuji S, Onodera O. (2002). Expanded polyglutamine stretches form an 'aggresome'. Neurosci Lett 323:215-218.

Tokatlidis K, Dhurjati P, Millet J, Beguin P, Aubert JP. (1991). High activity of inclusion bodies formed in Escherichia coli overproducing Clostridium thermocellum endoglucanase D. FEBS Lett 282:205-208.

Upadhyay AK, Murmu A, Singh A, Panda AK. (2012). Kinetics of inclusion body formation and its correlation with the characteristics of protein aggregates in Escherichia coli. PLoS One 7:e33951.

Vazquez E, Corchero JL, Burgueno JF, Seras-Franzoso J, Kosoy A, Bosser R, Mendoza R, Martinez-Lainez JM, Rinas U, Fernandez E, Ruiz-Avila L, Garcia-Fruitos E, Villaverde A. (2012). Functional inclusion bodies produced in bacteria as naturally occurring nanopills for advanced cell therapies. Adv Mater 24:1742-1747.

Wang Y, Meriin AB, Costello CE, Sherman MY. (2007). Characterization of proteins associated with polyglutamine aggregates: a novel approach towards isolation of aggregates from protein conformation disorders. Prion 1:128-135.

Wigley WC, Fabunmi RP, Lee MG, Marino CR, Muallem S, DeMartino GN, Thomas PJ. (1999). Dynamic association of proteasomal machinery with the centrosome. J Cell Biol 145:481-490.

Worrall DM, Goss NH. (1989). The formation of biologically active beta-galactosidase inclusion bodies in Escherichia coli. Aust J Biotechnol 3:28-32.

Zhang X, Qian SB. (2011). Chaperone-mediated hierarchical control in targeting misfolded proteins to aggresomes. Mol Biol Cell 22:3277-3288. 


\section{Figure captions}

Fig 1 GLA kinetics production in transiently transfected HEK 293F cells. Samples were collected 3, 4 and 5 days post-transfection, and processed as indicated in "Material and Methods" section in order to separate the soluble, secreted ("Extracel") fraction from the insoluble ("Ins") and soluble ("Sol") intracellular protein forms. Samples from non-transfected 293F cells taken at day 5 (labelled as "Mock cells, 5d") were treated with the same protocol and included as a negative control. Legend: MW: molecular weight marker. C+: positive control (purified, soluble GLA)

Fig 2 TEM images of HEK 293F cells transfected with pOpinE-GLA expression vector. Images correspond to cells before transfection (panels a, b), 3 days (panels c, d) and 5 days (panels e, f) after transfection. White arrows point out individual aggresomes. In panels $2 \mathrm{~d}$ and $2 \mathrm{f}$, closer views allow to distinguish the spherical building blocks that compose GLA aggresomes

Fig 3 SEM images of purified GLA aggresomes. Panel a: SEM image of aggresomes purified with "acetic acid disrupting buffer" from cells harvested 5 days post-transfection. In panel b, a closer view allows to clearly distinguish the spherical aggresomes building blocks

Fig 4 Solubilization of GLA aggresomes. Spontaneous solubilization of GLA aggresomes was followed by measuring the remaining enzymatic activity in the aggresome fraction (panel a) and the simultaneous increase of enzymatic activity in the supernatant (panel b). Similar results were obtained when following solubilization by estimating the GLA protein amount in the aggresome fraction (panel c) and in the supernatant (paneld). Data shown represents mean +/SEM of 4 independent samples tested each in duplicate (total $n=8)$

Fig 5 Stability and reusability of GLA aggresomes. Panel a: Stability of GLA aggresomes (black circles) versus soluble, secreted GLA (white circles), was followed by measuring the remaining enzymatic activity of both materials when incubated in acetic acid buffer, at $37^{\circ} \mathrm{C}$, for the indicated time periods. Panel b: Reusability was evaluated by performing successive enzymatic activity measurements ("rounds"), and further comparison with "Round 1" (activity defined as

$100 \%)$. For both types of experiments, data shown represents mean +/- SEM of 2 independent samples tested each in triplicate (total $n=6$ ) 